\title{
PERSPECTIVE
}

\section{Ethical Considerations of Fertility Preservation for Transmasculine and Nonbinary Youth}

\author{
Leila J. Katabi, ${ }^{1,2, *}$ Henry H. Ng, ${ }^{3,4}$ Carl G. Streed Jr., ${ }^{5,6}$ and Kavita S. Arora ${ }^{1,7}$
}

\begin{abstract}
An increasing number of young adolescents who identify as transgender or nonbinary are presenting to the health care system for gender affirmation therapy before the full progression of puberty. Gender-affirming therapy may impair future fertility, but options exist for fertility preservation. This perspective reviews these options for transmasculine and nonbinary youth, and explores related ethical considerations. The authors support the right of transgender and nonbinary youth to utilize available reproductive technologies, provide recommendations for treating health professionals, and advocate for increased research efforts and tools to aid patient decision making.
\end{abstract}

Keywords: adolescents; ethics; fertility preservation; infertility; transgender

\section{Introduction}

Among the $\sim 1.4$ million transgender adults in the United States, ${ }^{1}$ hormone therapy remains the most common component of gender-affirming care. ${ }^{2}$ With the number of adolescents identifying as transgender and nonbinary rising, ${ }^{3}$ individuals likely to receive hormone therapy as a component of gender-affirming care are also expected to grow. ${ }^{4}$ For transgender or nonbinary patients who seek care before the full progression of puberty, hormone therapy consists of gonadotropin releasing hormone $(\mathrm{GnRH})$ analogue to delay puberty, followed by gender-affirming hormones to allow the expression of secondary sex characteristics of the desired gender identity. ${ }^{5}$ Little is known about longterm effects of hormone therapy on future fertility, so authoritative guidelines recommend discussing potential infertility and fertility preservation with adolescent patients before initiating hormone therapy. ${ }^{5,6}$ This perspective reviews the fertility preservation options for adolescent transgender and nonbinary patients designated female at birth and explores related ethical considerations.

\section{Fertility Preservation Options}

Adolescent transgender and nonbinary patients designated female at birth can utilize two fertility preservations options without a partner-experimental ovarian tissue cryopreservation or mature oocyte cryopreservation. ${ }^{7}$ Prepubescent patients can only utilize ovarian tissue cryopreservation, an experimental procedure that involves harvesting ovarian tissue for cryopreservation. ${ }^{8}$ Live pregnancies have only been achieved from tissue transplanted back into the original donor, which would require cessation of gender-affirming hormone therapy in adulthood to allow the reimplantation of the ovary. Although unwanted female secondary sex characteristics would develop, some patients may accept these body changes if they identify more as nonbinary, want to gestate the pregnancy themselves, or value genetic parenthood more than changes in physical characteristics. ${ }^{9}$ Alternatively, there have been reports of live births conceived from transplanting fresh ovarian tissue to a related donor. ${ }^{8}$ Other areas of active research include harvesting immature oocytes with in vitro maturation before cryopreservation or

${ }^{1}$ Department of Bioethics, Case Western Reserve University, Cleveland, Ohio, USA.

${ }^{2}$ School of Medicine, Case Western Reserve University, Cleveland, Ohio, USA.

${ }^{3}$ Center for LGBT Health, Cleveland Clinic Foundation, Cleveland, Ohio, USA

${ }^{4}$ Public Health and Science Department, Baldwin Wallace University, Berea, Ohio, USA

${ }^{5}$ Department of Medicine, Boston University School of Medicine, Boston, Massachusetts, USA.

${ }^{6}$ Center for Transgender Medicine and Surgery, Boston Medical Center, Boston, Massachusetts, USA.

${ }^{7}$ Department of Obstetrics and Gynecology, MetroHealth Medical Center, Cleveland, Ohio, USA.

*Address correspondence to: Leila J. Katabi, MA, Department of Bioethics, Case Western Reserve University, 2109 Adelbert Road, Cleveland, OH 44106, USA, E-mail: ljk49@case.edu 
in vitro maturation of oocytes from previously preserved ovarian tissue. ${ }^{8}$ By the time patients reach child-bearing age, the latter may provide another avenue for utilizing cryopreserved tissue. Regardless of the method chosen, there are surgical and anesthetic risks without guaranteed future fertility. ${ }^{8}$

Since ovarian tissue cryopreservation requires surgical removal of the ovarian tissue, some dismiss this option due to recommendations against permanent surgery in minors. ${ }^{10}$ Rather than applying a blanket policy against this option, it is important to remember that there are no alternative options for adolescents who would be psychologically and physically harmed by progressing through puberty, yet wish to preserve fertility potential. Although experimental, it is currently offered to other adolescent patients (e.g., in oncology). ${ }^{8}$

A second option is mature oocyte cryopreservation, which requires forgoing $\mathrm{GnRH}$ analogue therapy to allow maturation of oocytes through puberty. The resulting changes to physical characteristics would preclude some, but not all, patients from considering this option, ${ }^{9}$ and some patients may experience significant gender dysphoria from required vaginal ultrasounds. ${ }^{7}$ Although there are risks associated with the hormone regimen and oocyte procurement without guaranteed future fertility, risks are well established and this method is not experimental. ${ }^{11}$ Furthermore, a recent case series demonstrated that this technique can be safely performed in transgender adolescents. ${ }^{12}$

Patients who utilize either option can subsequently gestate the pregnancy themselves, transfer gametes to a partner, or use a surrogate, ${ }^{13}$ depending on state laws.

\section{The Right to Utilize Fertility Preservation}

Robertson coined the term "procreative liberty" as the "freedom to decide whether or not to have offspring and to control the use of one's reproductive capacity." He argued that given its importance, procreative liberty should be given presumptive priority when faced with conflicting interests. ${ }^{14}$ Although not all individuals have reproductive capacity, just as is the case for cisgender youth, some transgender and nonbinary youth desire genetic parenthood. ${ }^{13,15}$ Access to fertility preservation technologies is a negative right in many countries. This means that society does not have an ethical obligation to help individuals fulfill their procreative liberty through insurance coverage, but must ensure that the right to reproduce (or not) is not interfered with by outside entities.
However, this view of reproduction as a negative right is changing and insurance policies are beginning to include fertility procedures. ${ }^{9}$ When viewed through the lens of reproductive justice (or the "human right to maintain personal bodily autonomy, have children, not have children, and parent the children we have in safe and sustainable communities"), availability and access to fertility preservation are an essential component of uplifting the needs of a marginalized population. Although arguing that procreative liberty should be a positive right is outside the scope of this perspective, at the least, transgender and nonbinary patients should be among the beneficiaries of these changes 9 and should not be barred from accessing services on the basis of gender identity. ${ }^{16}$

Furthermore, fertility preservation is supported by contemporary adolescent decision-making frameworks, such as the best interest standard, harm prevention model, and autonomy-based models. For the latter, Feinberg argued that minors have the right to an open future to protect their ability to make significant life choices as adults. ${ }^{17}$ As has been argued in other adolescent contexts, ${ }^{18}$ offering fertility preservation to transgender and nonbinary adolescent patients offers more of an open future than otherwise could be maintained. ${ }^{9}$

\section{Enhancing the Informed Consent Process}

Shared decision making should augment the process of informed consent when discussing fertility preservation because it is a high-risk decision with multiple medically reasonable options. This requires medical providers to explore patients' values to make meaningful recommendations, as exemplified in a recent article that demonstrated how differing values led two transgender adolescents to pursue opposite decisions regarding fertility preservation. ${ }^{19}$ Religious and cultural values, in particular, may impact decision making, though addressing this particularity is beyond the scope of this perspective.

Another important aspect of shared decision making is adequately relaying information to patients, which requires medical providers to have updated knowledge of treatment options. ${ }^{7}$ Unfortunately, physicians' selfreported limited knowledge of fertility preservation for transgender patients was recently identified as a barrier to care. ${ }^{20}$ To better support medical providers, a "decision tool web portal" that houses up-to-date literature and helps providers tailor patient discussions can be developed similar to what has been done for 
fertility preservation in oncology patients. ${ }^{21}$ The development of a structured protocol for counseling transgender adolescents about fertility preservation has also been proposed. ${ }^{22}$ This could help providers navigate discussions and promote the dissemination of accurate information.

Supporting the adolescent patient through the informed consent process is another important component of shared decision making. Although parents will retain ultimate decision-making authority due to legal and practical constraints, adolescent patients should be intimately involved in fertility discussions and their assent should be solicited. Adolescents may struggle to think abstractly about the future and balance their immediate goals with long-term consequences, so it is important to help them understand (1) potential effects of hormone therapy on their ability to have genetically related children; (2) that pursuing fertility preservation may postpone hormone therapy, causing prolonged distress or development of unwanted secondary sex characteristics; (3) specific procedural techniques and associated pain and discomfort; and (4) associated costs. The development of tools similar to what has been done for fertility preservation in oncology patients, such as a patient navigator, hotline, and patient-facing website, ${ }^{21}$ could further support patients.

It is also important for patients to discuss their feelings separate from family member to minimize potential familial pressure. Ideally, the health professional, patient, and family would agree upon the treatment plan, with the patient's values guiding the final decision. If disagreement occurs, standard mechanisms for conflict resolution, such as consulting support services (ethics, social work, and legal), clinical mediation, or revisitation of the discussion at a later time, are reasonable strategies to deal with the conflict.

\section{Addressing Knowledge Gaps}

Fertility issues for transgender and nonbinary youth are understudied, ${ }^{22}$ hindering informed consent. Little is known about the amount or duration of hormone exposure that could permanently impair fertility. ${ }^{10,23}$ Better understanding these consequences could influence patient decision making and provider recommendations. In addition, there are few studies about the parenting desires of transgender and nonbinary adolescents. ${ }^{15,24}$ Further exploring common concerns about parenting may help medical providers improve informed consent discussions and demonstrate the
Table 1. Summary of Recommendations for Health Professionals Treating Transgender and Nonbinary Adolescent Patients

Universally counsel adolescent patients about fertility preservation options before initiating hormone therapy.

Make accommodations for adolescent transgender and nonbinary patients in fertility clinics and include transgender-specific information on practice websites.

Utilize the shared decision-making model during the informed consent process and make a personalized recommendation for each patient based on their values and goals of care, as well as the appropriate medical considerations.

Help the adolescent patient understand (1) the potential effects of hormone therapy on their ability to have genetically related children; (2) that pursuing fertility preservation may postpone hormone therapy, causing prolonged distress or development of unwanted secondary sex characteristics; (3) specific procedural techniques and associated pain and discomfort; and (4) associated costs.

Discuss the adolescent patient's view of fertility preservation separate from other family members to minimize familial pressure.

Solicit the adolescent patient's desires based upon the information presented.

need for the development of technologies for adolescent transgender and nonbinary patients. Furthermore, studies have found low utilization rates of fertility preservation services by adolescent transgender patients. ${ }^{23,24}$ Cost has been identified as one barrier to care. ${ }^{3,23}$ It is also possible that although fertility clinics are increasingly making efforts to accommodate LGBTQ patients, ${ }^{25,26}$ lack of transgenderspecific information on fertility clinic websites could be negatively impacting the use of fertility preservation services by transgender and nonbinary adolescents. ${ }^{27}$ Ultimately, existing obstacles to care must be further elucidated and addressed to increase utilization rates and ensure equality in access.

The field of fertility preservation is advancing research for adolescent oncology patients far beyond that which is occurring for transgender and nonbinary patients. To respect the principle of justice and to better treat these patients, increased research efforts are necessary. This will require more transgender and nonbinary youth to participate in research, and although they should be considered a vulnerable population, precautionary thresholds should not be so stringent that they prevent scientific advancement. ${ }^{28}$

\section{Conclusion}

Fertility preservation for transgender and nonbinary youth balances the ethical considerations of autonomy, nonmaleficence, beneficence, and justice and is appropriate when viewed through both a reproductive justice and adolescent decision-making framework. Procreative liberty and the right to an open future lend support 
to transgender and nonbinary youths' right to utilize experimental ovarian tissue or mature oocyte cryopreservation. Research into fertility preservation in this patient population is lacking and should be promoted. Further knowledge, as well as the development of decision-making aids and standardized protocols, will contribute greatly to our ability to comprehensively care for transgender and nonbinary patients who present for care before the full progression of puberty.

\section{Disclaimer}

This article is solely the responsibility of the authors and does not necessarily represent the official views of the NIH.

\section{Author Disclosure Statement}

No competing financial interests exist.

\section{Funding Information}

K.S.A. is funded by the Clinical and Translational Science Collaborative of Cleveland, KL2TR000440, from the National Center for Advancing Translational Sciences (NCATS) component of the National Institutes of Health and NIH roadmap for Medical Research.

\section{References}

1. Flores A, Herman JL, Gates GJ, et al. How Many Adults Identify as Transgender in the United States? Los Angeles, CA: The Williams Institute, 2016.

2. James SE, Herman, JL, Rankin S, et al. The Report of the 2015 U.S. Transgender Survey. Washington, DC: National Center for Transgender Equality, 2016.

3. Bearelly $\mathrm{P}$, Rague JT, Oates RD. Fertility preservation in the transgender population. Curr Sex Health Rep. 2020;12:40-48.

4. Streed CG, Jr., Hedian HF, Bertram A, et al. Assessment of internal medicine resident preparedness to care for lesbian, gay, bisexual, transgender, and queer/questioning patients. J Gen Intern Med. 2019; 24:893-898.

5. Hembree WC, Cohen-Kettenis PT, Gooren L, et al. Endocrine treatment of gender-dysphoric/gender incongruent persons: an endocrine society clinical practice guideline. J Clin Endocrinol Metab. 2017;102:3869-3903.

6. Coleman E, Bockting W, Botzer M, et al. Standards of care for the health of transsexual, transgender, and gender nonconforming people, version 7 . Int J Transgenderism. 2012;13:165-232.

7. Finlayson $C$, Johnson EK, Chen D, et al. Proceedings of the working group session on fertility preservation for individuals with gender and sex diversity. Transgender Health. 2016;1:99-107.

8. Pfeifer S, Goldberg J, Lobo R, et al. Ovarian tissue cryopreservation: a committee opinion. Fertil Steril. 2014;101:1237-1243.

9. Normand S. Fertility preservation in transgender and gendernonconforming youth and adolescents. In: Reproductive Ethics: New Challenges and Conversations. (Campo-Engelstein L, Burcher P; eds). Cham, Switzerland: Springer, 2017, pp. 77-84.

10. Jarin J, Johnson E, Gomez-Lobo V. Fertility preservation in patients with gender dysphoria. In: Pediatric and Adolescent Oncofertility. (Woodruff T, Gosiengfiao Y; eds). Cham, Switzerland: Springer, 2017, pp. 55-67.
11. Pfeifer S, Goldberg J, McClure D, et al. Mature oocyte cryopreservation: a guideline. Fertil Steril. 2013;99:37-43.

12. Chen D, Bernardi LA, Pavone ME, et al. Oocyte cryopreservation among transmusculine youth: a case series. J Assist Reprod Genet. 2018;34: 2057-2061.

13. Eyler EA, Pang SC, Clark A. LGBT assisted reproduction: current practice and future possibilities. LGBT Health. 2014;1:151-156.

14. Robertson, JA. Children of Choice: Freedom and the New Reproductive Technologies. Princeton, NJ: Princeton University Press, 1996.

15. Strang JF, Jarin J, Call D, et al. Transgender youth fertility attitudes questionnaire: measure development in nonautistic and autistic transgender youth and their parents. J Adolesc Health. 2018;62: 128-135.

16. Daar J, Amato P, Benward J, et al. Access to fertility services by transgender persons: an Ethics Committee opinion. Fertil Steril. 2015; 104:1111-1115.

17. Feinberg J. Freedom and Fulfillment: Philosophical Essays. Princeton, NJ: Princeton University Press, 1992.

18. Jadoul $P$, Dolmans MM, Donnez J. Fertility preservation in girls during childhood: is it feasible, efficient and safe and to whom should it be proposed? Hum Reprod Update. 2010;16:617-630.

19. Chen D, Simons L. Ethical considerations in fertility preservation for transgender youth: a case illustration. Clin Pract Pediatr Psychol. 2018;6: 93-100.

20. Tishelman AC, Sutter ME, Chen D, et al. Health care provider perceptions of fertility preservation barriers and challenges with transgender patients and families: qualitative responses to an internal survey. J Assist Reprod Genet. 2019;36:579-588.

21. Woodruff TK. Oncofertility: a grand collaboration between reproductive medicine and oncology. Reproduction. 2015;150:S1-S10.

22. Nahata L, Chen D, Moravek MB, et al. Understudied and under-reported: fertility issues in transgender youth-A narrative review. J Pediatr. 2019; 205:265-271.

23. Chen $D$, Simons $L$, Johnson EK, et al. Fertility preservation for transgender adolescents. J Adolesc Health. 2017;61:120-123.

24. Nahata L, Tishelman AC, Caltabellotta NM, Quinn GP. Low fertility preservation utilization among transgender youth. J Adolesc Health. 2017;61: 40-44.

25. Soares SR, Cruz M, Vergara V, et al. Donor IUI is equally effective for heterosexual couples, single women and lesbians, but autologous IUI does worse. Hum Reprod. 2019;34:2184-2192.

26. Monseur BC, Franasiak JM, Sun $L$, et al. Double intrauterine insemination (IUI) of no benefit over single IUI among lesbian and single women seeking to conceive. J Assist Reprod Genet. 2019;36:20952101.

27. Wu HY, Yin O, Monseur B, et al. Lesbian, gay, bisexual, transgender content on reproductive endocrinology and infertility clinic websites. Fertil Steril. 2017;108:183-191.

28. Fisher CB, Mustanski B. Reducing health disparities and enhancing the responsible conduct of research involving LGBT youth. Hastings Cent Rep. 2014;44:S28-S31.

Cite this article as: Katabi LJ, Ng HH, Streed CG, Arora KS (2020) Ethical considerations of fertility preservation for transmasculine and nonbinary youth, Transgender Health 5:4, 201-204, DOI: 10.1089/ trgh.2020.0004.

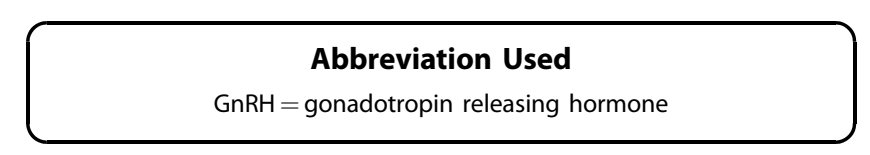

